\title{
Circulating mRNA Lamin B1 for Detection of Early Stages of Hepatocellular Carcinoma
}

Nasser Sadek Rezk ${ }^{1}$, Mona Mostafa Osman ${ }^{1}$, Amira Ibrahim Hamed ${ }^{1}$, Ashraf Mohammad AlBreedy $^{2}$, Hoda Ahmed Abd El - Sattar ${ }^{1}$, and Amani Mohamed A. Ghani. ${ }^{1}$

1Clinical Pathology Department, Faculty of Medicine, Ain Shams University.

2Department of Tropical Medicine and Infectious diseases, Faculty of Medicine, Ain Shams University

Correspondence: $\underline{\text { dr_amani83@hotmail.com }}$

\begin{abstract}
Background: Hepatocellular carcinoma (HCC) is the fifth most common cancer and the third leading cause of death worldwide, causing 600,000 deaths annually. In Egypt, HCC accounts for $4.7 \%$ of all liver diseases. Hospital-based studies have reported an overall increase in the relative frequency of all liver cancers in Egypt from approximately $4 \%$ in 1993 to $7.3 \%$ in 2003 , more than $95 \%$ of which being HCC. Patients diagnosed at an early stage of HCC have the best prognosis, where resection and transplantation achieve the best outcomes in well-selected candidates, with a 5 -year-survival rate of 50 to $70 \%$. In this regard, the present study aimed to demonstrate the potential clinical importance of plasma levels of circulating mRNA of lamin B1 by real-time RT PCR as an early marker of HCC..
\end{abstract}

Methods: This study was conducted at the Clinical Pathology Department and the subjects were selected from Department of Tropical Medicine and Infectious Diseases at Ain Shams University Hospitals. It included thirty (30) HCC patients of different stages (group I), they were further divided into 3 stages using BCLC staging system into stage $A, B$ and $C$, in addition to ten (10) cases with benign liver diseases serving as patient controls (group II) and ten (10) healthy age-and sex-matched subjects serving as healthy controls (group III).

Results: The plasma laminB1 mRNA showed a much better performance to differentiate between HCC cases and the 2 control groups, where its sensitivity was $100 \%$ and specificity was $90 \%$.

Conclusions: Addition of plasma lamin B1 mRNA to the current standard tests for diagnosis of HCC as a new diagnostic and screening tool. This, in turn, could greatly improve the ability to identify such patients and thus could allow them to benefit from earlier treatment.

Keywords: circulating mRNA - early HCC $\cdot B C L C$ staging system

\section{Background}

Hepatocellular carcinoma is one of the most common and aggressive cancers, being the third cancer causing death worldwide. Its incidence has increased sharply in the last 5-10 years, by 3\% - 9\% annually, among the patients with chronic liver diseases.

The high mortality associated with this disease is mainly attributed to the inability to diagnose HCC patients at an early stage. In Egypt, HCC accounts for $4.7 \%$ of all liver diseases ${ }^{1}$. The current approach to screen for the presence of $\mathrm{HCC}$ in high-risk populations is the combination of serum alpha-fetoprotein (AFP) and ultrasonography. However, elevated serum AFP is only observed in about $60 \%-70 \%$ of HCC patients and, to a lesser extent (33\%), in patients with smaller-sized HCCs.

Moreover, the non-specific elevation of serum AFP in $15 \%-58 \%$ of patients with chronic hepatitis and $11 \%$ $47 \%$ of patients with liver cirrhosis have led to the exclusion of AFP as a single screening test for HCC patients in the European association for the study of the 
liver (EASL) guidelines ${ }^{2}$.

Currently, Ultrasonography (US) represents the primary radiologic tool for HCC surveillance of at-risk populations. However, US has limited sensitivity $<$ $60 \%$ ) to detect small HCC especially in obese patients and those with underlying cirrhosis. The gold standard test for diagnosis of HCC is spiral computerized tomography (C.T.) which has advanced sensitivity $(80 \%)$ and specificity (96\%). Unfortunately, its use in HCC surveillances have been limited, as it was reported that the diagnostic efficacy of CT is diminished in small tumors (less than $2 \mathrm{~cm}$ ) owing to the hypovascularization of small-sized tumors. Also, it requires administration of contrasting materials to patients which may be potentially harmful and it is not available in many medical centers ${ }^{3,4}$.

Lamin B1 is an intermediate filament protein that forms a network lining the inner nuclear membrane; it forms the major component of nuclear lamina which provides structural support of the nuclear envelope. It is involved in controlling gene expression via the organization of chromatin, deoxyribonucleic acid (DNA) replication, repair and transcription ${ }^{5}$. Lamin B1 was also found to control oxidative stress. It works as transcriptional co-regulator whose dysregulation may initiate cancer signaling processes. Lamin B1 was evaluated in liver tissue samples of HCC patients using immunohistochemistry and it was found to be highly expressed in those patients in comparison to cirrhotic patients. Lamin B1 mRNA was also evaluated in plasma and showed an elevation in the plasma of HCC patients ${ }^{6}$.

Finding new biomarkers that have higher sensitivity and specificity for the early diagnosis of HCC among high risk patients, would have a great impact on the disease. The present study aimed at demonstrating the potential clinical role of plasma levels of mRNA of lamin B1 by real-time RT PCR as an early marker of HCC. Also, to correlate its levels with tumor size and number of nodules represented by Barcelona-Clinic Liver Cancer (BCLC) Staging System, as well as, with serum AFP.

\section{Methods Study design}

This study was conducted at the Clinical Pathology Department in collaboration with the Department of Tropical Medicine and Infectious Diseases at Ain Shams University Hospitals. It included thirty (30) HCC patients of different stages, in addition to ten (10) cases with benign liver diseases serving as patient controls and ten (10) healthy subjects age and sex matched serving as healthy controls. A verbal consent was taken from all participants sharing in the study. The study protocol was approved by the Researcher
Ethics Committee at faculty of Medicine, Ain shams university. Individuals in this study were classified into:

1-Group I (HCC Group): This included 30 patients with primary HCC on top of viral cirrhosis (all were HCV positive). The diagnosis was confirmed by spiral CT. They were further divided into 3 stages according to the tumor size and tumor number using BCLC staging system:

a- Stage A $(\mathrm{n}=14)$ : (single tumor, $\leq 3 \mathrm{~cm})$.

b- Stage B $(\mathrm{n}=7)$ : (2 tumor nodules, $>3 \mathrm{~cm})$.

c- $\quad$ Stage $C(n=9)$ : (multinodular, $>3 \mathrm{~cm})$.

2-Group II (Pathological Control Group): This included 10 patients age and sex matched with benign hepatic disorders namely, chronic hepatitis > 6 months and liver cirrhosis. The diagnosis was based on the clinical picture, ultrasonography (for exclusion of HCC), elevated aspartate aminotransferase (AST) or alanine aminotransferase (ALT), low albumin and prolonged prothrombin time (PT).

3- Group III (Healthy Control Group): This included 10 age and sex-matched healthy individuals

All individuals included in this study were subjected to the following:

1- Full history taking focusing on previous hepatic disorders or predisposing factors preceeding liver disease.

2- Thorough clinical examination, with special emphasis on abdominal examination, jaundice, oedema and ascitis.

3- Radiological investigations including CT scan (for HCC patients only), abdominal ultrasound for patients with hepatic disorders and normal controls.

4- Laboratory investigations including:

a. Routine liver function tests, namely AST, ALT, albumin, total bilirubin, direct bilirubin, INR, prothrombin time (P.T) \& complete blood count (CBC). b. Serum alphafetoprotein (AFP) was assayed by chemiluminescent-immunometric technique.

c. Plasma mRNA Lamin B1 was quantitated by Realtime RT-PCR.

Quantitative Assay of Lamin B1 mRNA by Realtime RT-PCR:

This process was performed through several steps including RNA extraction, reverse transcription (RT) and cDNA synthesis and finally DNA amplification and detection by Real-Time PCR.

a-RNA extraction: RNA extraction was performed on the EDTA-K3 plasma samples. The plasma was aliquoted and stored at $-80^{\circ} \mathrm{C}$ till assay which was done by miRNeasy Mini Kit provided by Qiagen.

b-Real-time RT and cDNA synthesis: Two-step (RT)c DNA synthesis was performed on the extracted RNA prepared in the previous step using QuantiTect reverse transcription kit provided by Qiagen.

c- DNA amplification and detection by Real-Time RT PCR: Real-Time two-step RT-PCR master mix reagents were provided by Qiagen. The PCR plate was placed in the real-time cycler and the program was 
started. 45 cycles were done. Data analysis was performed. Results were reported in relative quantification. Relative quantification determines the changes in mRNA levels of a gene across multiple samples and expresses its relative levels to the levels of an internal control RNA (housekeeping gene), in our study, GAPDH was used. Finally, the normalized level of target gene expression was calculated by using the formula: $2-\Delta \Delta \mathrm{Ct}$.

\section{Statistical methods:}

Data analysis was done using IBM SPSS statistics (V. 22.0, IBM Corp., USA, 2013). Data were expressed as mean \pm standard deviation (SD) for quantitative parametric measures. Skewed data were expressed as median and inter-quartile range (IQR) which extends between the $25^{\text {th }}$ and $75^{\text {th }}$ percentiles $\left(Q_{1}\right.$ and $Q_{3}$, respectively). Significance Tests as Kruskal-Wallis test and Mann-Whitney U-test were done. Spearman's rank correlation coefficient $\left(\mathrm{r}_{\mathrm{s}}\right)$ was used.

\section{Results:}

A statistically significant difference was found among the three studied groups as regards AFP and lamin B1, the median levels of lamin B1 revealed statistically significant difference among group I versus II and group I versus III respectively, but no significant difference between group II versus III (table 1).

Comparative studies between the 3 stages of HCC patients are demonstrated in the table (2) below. A statistical significant increase was found as regards, AFP and lamin B1 $(\mathrm{H}=9.9, \mathrm{p}<0.01 \& \mathrm{H}=24.4$, $\mathrm{p}<0.001$, respectively).

In context with the tumor size and nodules'number comparison between HCC patients with one tumor nodule versus those with 2 or multiple tumor nodules revealed that the first group was statistically significantly different from the second group as regards AFP and lamin B1. Moreover, comparison between HCC patients with tumor size $>3 \mathrm{~cm}$ versus those with tumor size $\leq 3 \mathrm{~cm}$ as regards, AFP and lamin B1 revealed a statistically significant difference between the two groups.

A significant positive correlation between AFP and lamin B1, was shown only in group I, but not in group II nor III.

ROC curve analysis was applied to the current study results to examine the diagnostic performance of lamin B1\& AFP as tumor markers in HCC at different cut-off values. At a cut-off level of $5.0 \mathrm{ng} / \mathrm{dL}$, the diagnostic performance of AFP for differentiation between HCC cases and the 2 control groups was $80 \%$ sensitivity, 90\% specificity, $75 \%$ NPV, $92.3 \%$ PPV and $84 \%$ efficacy. Meanwhile, the plasma laminB1 mRNA showed a much better performance to differentiate between HCC cases and the 2 control groups, where at cutoff $2^{-\Delta \Delta \mathrm{CT}}=1.4$, sensitivity was $100 \%$, specificity was $90 \%$, NPV was $100 \%$, PPV was $93.4 \%$ and efficacy was $96 \%$.

\section{Discussion:}

Hepatocellular carcinoma (HCC) is one of the most common malignancies worldwide and one of the major causes of death, because of its high frequency and poor prognosis. Conventional tests of liver function cannot distinguish HCC from other hepatic masses or from cirrhosis. Accordingly, diagnosis of HCC depends mainly on the detection of tumor mass by diagnostic modalities such as ultrasound, spiral CT and liver histopathology by biopsy, in addition to tumor markers in the sera of the HCC patients. Serum AFP concentrations have been shown to be the most useful tumor marker with regards to $\mathrm{HCC}$ but it may be normal in up to $40 \%$ of patients; lacking sensitivity. Moreover, it may be increased in hepatitis and cirrhotic patients; lacking specificity. The availability of a more sensitive serological marker that distinguishes between HCC and benign hepatic lesions would therefore, be very useful for early and specific diagnosis ${ }^{\mathbf{1}}$.

Table 1:

\begin{tabular}{|c|c|c|c|c|}
\hline \multirow{2}{*}{ Parameter } & $\begin{array}{l}\text { Groupl } \\
n=30\end{array}$ & $\begin{array}{l}\text { Group II } \\
n=10\end{array}$ & $\begin{array}{l}\text { Group } \\
\text { III } \\
n=10\end{array}$ & \multirow[t]{2}{*}{$\mathbf{p}$} \\
\hline & $\begin{array}{l}\text { Median } \\
\text { (Q1-Q3) }\end{array}$ & $\begin{array}{l}\text { Median } \\
\text { (Q1-Q3) }\end{array}$ & $\begin{array}{l}\text { Median } \\
\text { (Q1-Q3) }\end{array}$ & \\
\hline $\begin{array}{l}\text { AFP } \\
(n g / d L)\end{array}$ & $\begin{array}{l}31.6 \\
(6.6- \\
404.5)\end{array}$ & $\begin{array}{l}3.5 \\
(1.1-5.4)\end{array}$ & $\begin{array}{l}1.3 \\
(1.0-3.4)\end{array}$ & $<0.01$ \\
\hline $\begin{array}{l}\text { LaminB1 } \\
(2-\Delta \Delta c t)\end{array}$ & $\begin{array}{l}3.9 \\
(2.0-13.3)\end{array}$ & $\begin{array}{l}0.9 \\
(0.8-1.1)\end{array}$ & $\begin{array}{l}1.0 \\
(0.9-1.2)\end{array}$ & $<0.01$ \\
\hline
\end{tabular}

Table 2:

\begin{tabular}{|l|l|l|l|l|}
\hline & $\begin{array}{l}\text { Stage } \\
\text { A } \\
\mathbf{n}(14) \\
\text { Median } \\
\text { (Q1- } \\
\mathbf{Q 3 )}\end{array}$ & $\begin{array}{l}\text { Stage } \\
\mathbf{B} \\
\mathbf{n}(7) \\
\text { Median } \\
\text { (Q1- } \\
\mathbf{Q 3 )}\end{array}$ & $\begin{array}{l}\text { Stage C } \\
\mathbf{n}(9) \\
\text { Median } \\
\text { (Q1-Q3) }\end{array}$ & $\mathbf{p}$ \\
\hline $\begin{array}{l}\text { AFP } \\
\text { (ng/dL) }\end{array}$ & $\begin{array}{l}7.9 \\
(3.4-\end{array}$ & $\begin{array}{l}243 \\
(27.5-\end{array}$ & $\begin{array}{l}251 \\
(13.8-\end{array}$ & $<0.01$ \\
& $51.9)$ & $11793)$ & $24414.5)$ & \\
\hline $\begin{array}{l}\text { LaminB1 } \\
(\mathbf{2 -} \Delta \Delta \mathbf{c t})\end{array}$ & $\begin{array}{l}2.0 \\
(1.7-\end{array}$ & $\begin{array}{l}5.1 \\
(3.7-6)\end{array}$ & $\begin{array}{l}19.4 \\
(14.7-\end{array}$ & $<0.001$ \\
& $2.4)$ & & $28.2)$ & \\
& & & & \\
\hline
\end{tabular}


The current study revealed that a significant increase in the level of LMNB1 in the HCC patient group than in the cirrhotic and control groups. These results agreed with Sun et al. ${ }^{3}$, who reported that LMNB1 is a potential HCC marker that was detected even in the earliest stage of HCC; LMNB1 acts intracellularly or extracellularly in the form of protein and mRNA, respectively. The expression of this marker could be a result of lysis of cancer cells or encoded by tumorrelated genes ${ }^{3}$.

The plasma levels of lamin B1 $\left(2^{-\Delta \Delta c t}\right)$, was higher in late stages of $\mathrm{HCC}(\mathrm{B} \& \mathrm{C})$ compared to early stage $(\mathrm{A})$. This suggests that lamin B1 is correlated to increased invasiveness and promotes progression. This may be related to the fact that lamin B1 is present in active tumor development ${ }^{7}$.

The potential value of lamin B1 as an early diagnostic marker of HCC was demonstrated in the present study, where a statistically significant increase in plasma level among stage A HCC patients was observed compared to CLD patients. This is due to specific involvement of lamin B1 in carcinogenesis process since it increases in cancer cells more than cirrhotic cells ${ }^{8}$.

\section{References:}

1. El-Garem, H.; Abdel- Hafez, H.; Foaud, A.; Al Akel, W.; Atia, M. Salah, M.; Khatteb, H.; Osman, H.; Ragab, K. and Zayed, N. (2013): Tissue biomarkers in the early detection of hepatocellular carcinoma among egyptian patients with chronic hepatitis C: A possible genetic profile. British J. of Medicine \& Medical Research; 3(4): 1858-1870.

2. Bruix, J. and Sherman, M. (2011): American association for the study of liver diseases. Management of hepatocellular carcinoma: an update Hepatology; 53(3):1020-1022.

3. Sun, S.; Xu, M. Z.; Poon, R. T.; Day, P. J. and Luk, J. M. (2010): Circulating lamin B1 (LMNB1) biomarker detects early stages of liver cancer in patients. J. Proteome Res.; 9: 70-78.

4. Kim, C.K.; Lim, J.H. and Lee, W.J. (2011): Detection of hepatocellular carcinomas and dysplastic nodules in cirrhotic liver: accuracy of ultrasonography in transplant patients. J. Ultrasound Med.; 20: 99-104.

5. Dechat, T.; Shimi, T.; Adam, S. A.; Rusinol, A. E.; Andres, D. A.; Spielmann, H. P.; Sinensky, M. S. and Goldman, R. D. (2008): Alterations in mitosis and cell cycle progression caused by a mutant lamin A known to accelerate human aging. Proc. Natl. Acad. Sci. USA.; 104: 4955-4960.

6. Malhas, A.N.; Lee, C.F. and Vaux, D.J. (2009): Lamin B1 controls oxidative stress responses via Oct-1. J. Cell Biol.; 184(1): 45-55.

7. Wong, K-F. and Luk, J.M. (2012): Discovery of lamin B1 and vimentin as circulating biomarkers for early hepatocellular carcinoma. Djuro josic and Douglas C. Hixson (eds.), Liver proteomics: Methods and protocols, Methods in molecular biology; 909: 295-310.

8. Lim, S. O.; Park, S. J.; Kim, W.; Park, S. G.; Kim, H. J.; Kim, Y. I.; Sohn, T. S.; Noh, J. H. and Jung, G. (2002): Proteome analysis of hepatocellular carcinoma. Biochem. Biophys. Res. Commun.; 291 (4): 1031-1037 